УДК 550.831

ISSN 1609-0691

DOI: https://doi.org/10.17308/geology.2020.4/3127

Поступила в редакцию: 24.11.2020

Принята к публикации: 01.12.2020

Опубликована онлайн: 18.12 .2020

\title{
Вопросы обработки стационарных измерений гравиметрами
}

\author{
Ю. В. Антонов ${ }^{\bowtie}$, И. Ю. Антонова, И. А. Пономаренко \\ Воронежский государственный университет \\ Университетская пл.,1, 394018, Воронеж, Российская Федерация
}

\begin{abstract}
Аннотация
Введение: При сравнении пульсаций силы тяжести на станциях и обсерваториях различных континентов обнаружена синхронность колебаний. Часто при обработке оказывается, что измерения проведены с различной дискретностью. В таких случаях приходится проводить дискретизацию, чтобы измерения со всех станций имели один шаг дискретизации. Как правило, все измерения приравнивают к измерениям, где шаг дискретизации наибольший. Аналогичная ситуация возникает в случае, когда имеется переизбыток наблюдений, когда компьютеры не могут справиться с объемом вычислений. Тогда приходится проводить искусственно дискретизацию данных путем увеличения шага наблюдений. Дискретизация может проводиться или путем выборки отдельных значений через определенный интервал или путем вычисления среднеинтервального значения.

Методика: Оба способа дискретизации были опробованы на измерениях двухшарового сверхпроводящего гравиметра. Измерения данного гравиметра позволяют оценить не только выборку значений силы тяжести, но ее вертикального градиента. Гравиметр выбран из-за того, что является наиболее точным прибором на сегодняшний день. Измерения проводятся с дискретизацией в одну секунду, что позволяет искусственно изменять дискретизацию и оценивать влияние дискретизации на различного рода трансформации с наблюдениями. Исходные данные по измерениям указанного прибора взяты из общедоступных источников.

Результаты и обсуждение: Дискретизация с использованием осреднения сохраняет практически большую часть информации и должна использоваться при расчетах. Дискретизацию при простой выборке можно использовать преимущественно при качественной интерпретации результатов измерений.

Заключение: По результатам опробования рекомендуется использовать дискретизацию на основе среднеинтервального осреднения.

Ключевые слова: лунно-солнечные вариации силы тяжести; неприливные вариации силы тяжести; вертикальный градиент силы тяжести; метеорные потоки; пульсации.

Для цุитирования: Антонов Ю. В., Антонова И. Ю., Пономаренко И. А. Вопросы обработки стационарных измерений гравиметрами// Вестник Воронежского государственного университета. Ceрия: Геология. 2020. №4. С. 59-66. DOI: doi.org/10.17308/geology.2020.4/3127
\end{abstract}

\section{Введение}

В настоящее время во всём мире ведутся мониторинговые наблюдения геофизических полей на временных или постоянных станциях и обсерваториях. Одним из параметров записей является изменение гравитационного поля. К сожалению, единых правил к выбору аппаратуры для регистрации наблюдений нет. Не говоря обо всех требованиях к наблюдениям, оста- новимся лишь только на дискретизации записей. Как правило, все записи проводятся с различной дискретизацией. Это обстоятельство часто определяется техническими характеристиками приборов или целями, связанными с решением тех или иных задач. Нередко все эти записи начинают сравнивать между собой, не обращая никакого внимания на разность дискретизации в измерениях. Часто ряды наблюденных значений из-

Контент доступен под лицензией Creative Commons Attribution 4.0 License.

\footnotetext{
凶 Антонов Юрий Васильевич, e-mail: yuriyantonov@yandex.ru 
за слишком большого объема пытаются уменьшить. Bсе это, как показывают исследования, имеет существенное значение.

\section{Методика обработки и обсуждение результатов}

В конкретном случае остановимся на стационарных наблюдениях двухшарового сверхпроводящего гравиметра. Гравиметр выбран из-за того, что является наиболее точным прибором на сегодняшний день. Измерения проводятся с дискретизацией в одну секунду, что позволяет искусственно изменять дискретизацию и оценивать влияние дискретизации на различного рода трансформации с наблюдениями. Исходные данные по измерениям указанного прибора взяты из общедоступных источников $[1,2]$.

Обратимся к рис. 1. Здесь представлены синхронные измерения силы тяжести двумя датчиками. Внешне графики (рис. $1, a$ ) повторяют лунно-солнечные вариации силы тяжести. Дело в том, что неприливные вариации по интенсивности на порядок меньше лунно-солнечных вариаций. В этом есть своя польза [3]. Достаточно легко и просто с высокой надежностью определяется цена деления для обоих датчиков. Хотя в данном случае для демонстрации (рис. 1) взят временной отрезок, где неприливная вариация по амплитуде сопоставима с лунно-солнечной вариацией силы тяжести [4-6]. Но длительность этой пульсации не превышает трех суток, а весь сентябрь 2019 г. практически не подвержен пульсациям. Кстати, приведенная пульсация силы тяжести вызвана метеорным потоком Сентябрьскими Эпсилон Персеидами, который не отличается большим зенитным числом (количество метеоров в час), но в отдельные годы вызывают значительные пульсации, что вызвано наличием очень мелких частиц, не сгорающих в атмосфере.

Выбор в пользу двухшарового гравиметра объясняется также возможностью вычисления вертикального градиента силы тяжести. Вертикальный градиент определяется изменением силы тяжести по вертикали. Чтобы определить вертикальный градиент инструментально, достаточно измерить на двух высотах значение силы тяжести (в нашем случае 20 см), взять разность этих значений и разделить на разность высот. Несмотря на высокую точность измерений прибором, проблему сдвоенных гравиметров в данном случае решить не удалось. Хоть и небольшое смещение нуль-пункта, но в вычисленных значениях градиента наблюдается, т.е. можно судить лишь об относительных изменениях градиента. Но зато вычисление разности между показаниями датчиков ослабляет лунно-солнечные вариации силы тяжести других внешних воздействий.

Исходные данные по измерениям гравиметров (рис. $1, a$ ) были обработаны: вычислен вертикальный градиент и значения силы тяжести (рис.1, b). Пульсация силы тяжести отмечается как по градиенту, так и по значениям силы тяжести. Графики градиента и значений силы тяжести (рис. $1, b$ ) морфологически друг от

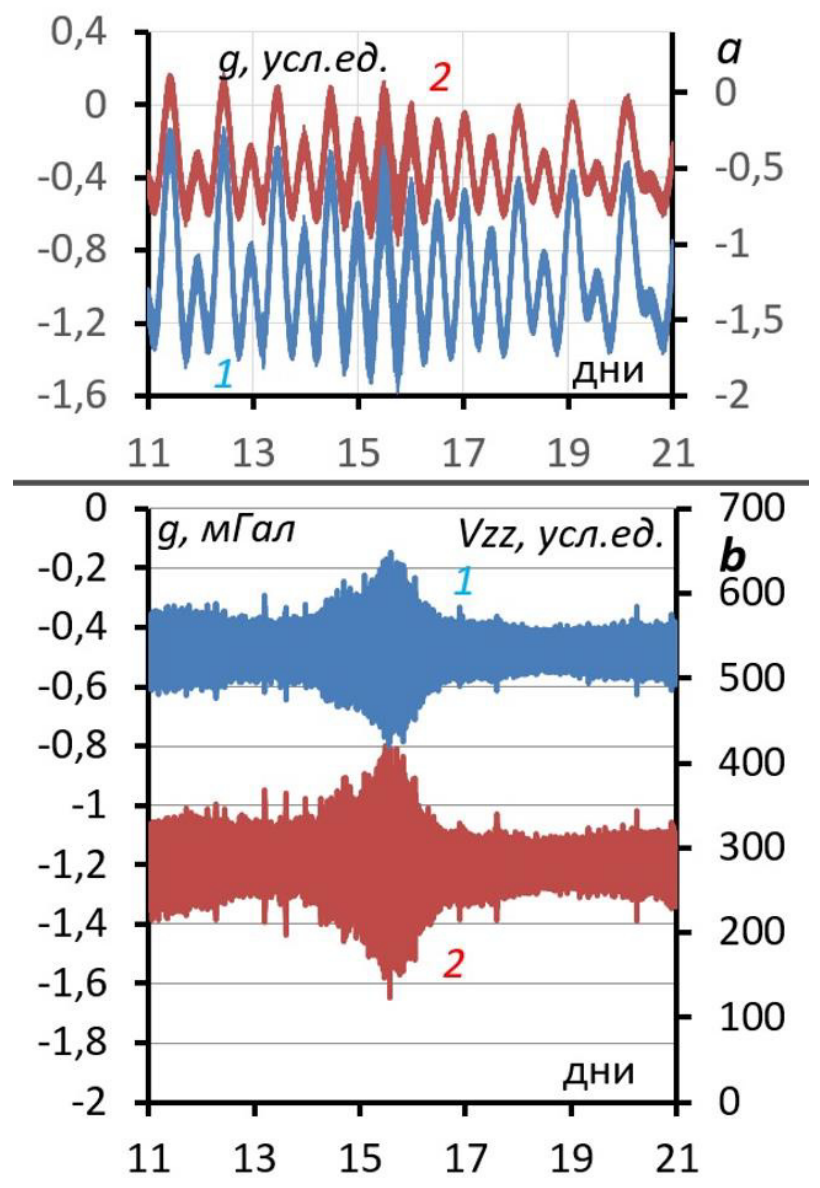

Рис. 1. Наблюденные значения с помощью двухшарового сверхпроводящего гравиметра на обсерватории BFO (Германия) 11-21 сентября 2019 г.: $a$ - исходные измерения силы тяжести, полученные с помощью датчиков №1 (кривая 1) и №2 (кривая 2); $b$ - вычисленные значения силы тяжести (кривая 2) и вертикального градиента силы тяжести (кривая 1).

[Fig. 1. Observed values using a two-step superconducting gravimeter at the BFO Observatory (Germany) on September 11-21, 2019.: $a$ - initial gravity measurements obtained using sensors no. 1 (curve 1) and no. 2 (curve 2); $b$ - calculated values of gravity (curve 2) and vertical gravity gradient (curve 1).]

друга почти не отличаются. А теперь представим, что наблюдения происходят с интервалом не через секунду, а с дискретностью 15, 30, 60 и 120 с. Проведем дискретную выборку через указанные интервалы (рис. 2). Полученные графики для всех указанных интервалов внешне ничем не отличаются от графиков на рис. $1, b$. Ту же саму операцию проделаем для тех же интервалов, но только с тем отличием, что для каждого интервала возьмем среднеинтервальное значение силы тяжести и градиента (рис. 3). Подобная операция осреднения при обработке долговременных рядов наблюдений проводится чаще всего. Различие между дискретной выборкой (рис. 2) и выборкой с осреднением (рис. 3) разительно.

С ростом интервала осреднения резко уменьшается амплитуда колебаний, что естественно должно проходить при осреднении [4-6]. Вместе с тем колебания небольшой амплитуды не исчезают, хотя по 


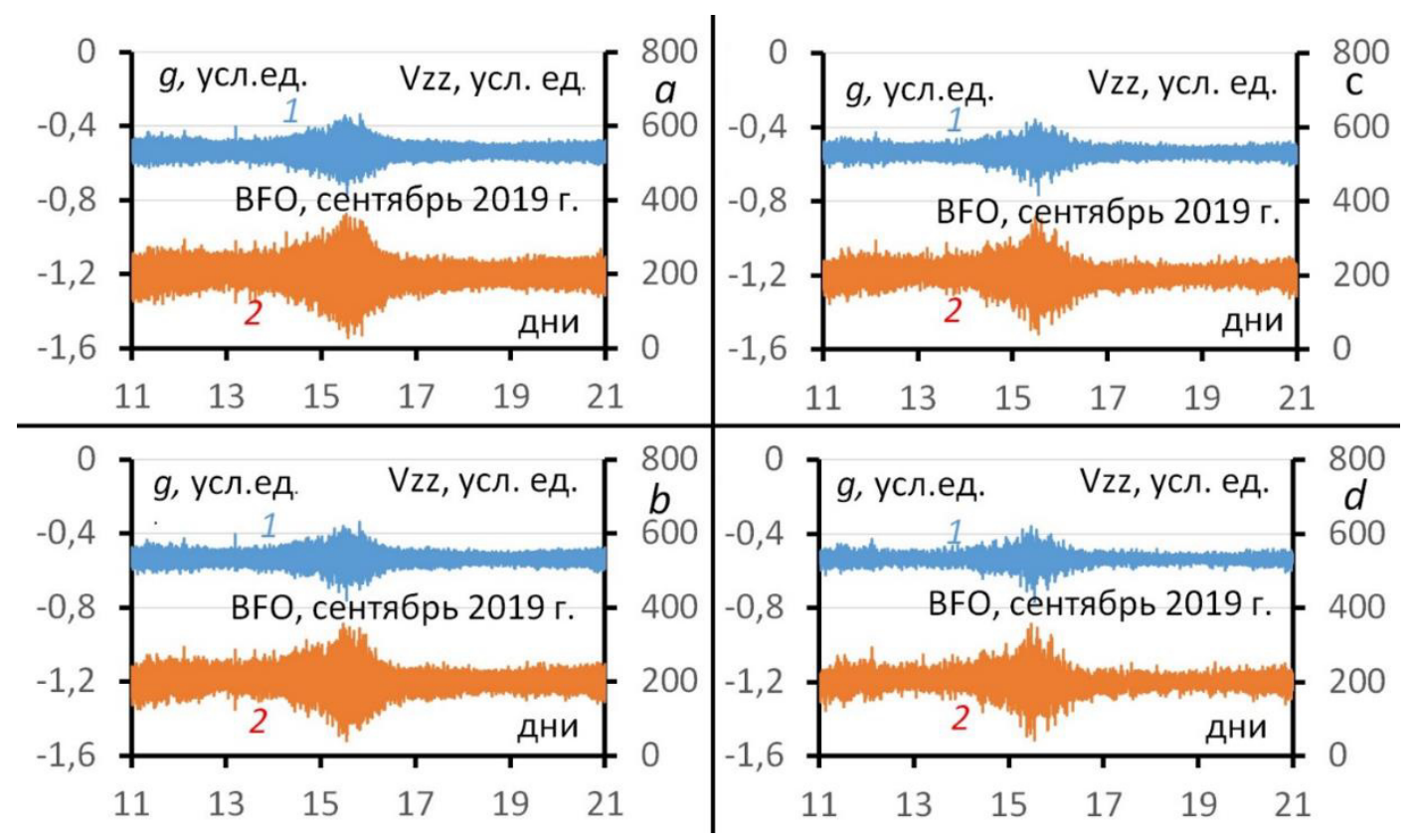

Рис. 2. Распределение силы тяжести и вертикального градиента, полученных с помощью дискретной выборки на интервалах $15,30,60$ и 120 секунд. $a$ - интервал выборки 15 с.: кривая 1 - значения вертикального градиента, кривая 2 - значения силы тяжести; $b, c, d$ - то же самое для интервалов $30,60,120$ с.

[Fig. 2. Distribution of gravity and vertical gradient obtained using a discrete sample at intervals of 15, 30, 60 and 120 seconds. $a-$ sampling interval of $15 \mathrm{~s}$.: curve 1 - values of the vertical gradient, curve 2 - values of gravity; $b, c, d$ - the same for intervals of $30,60,120 \mathrm{~s}$.]

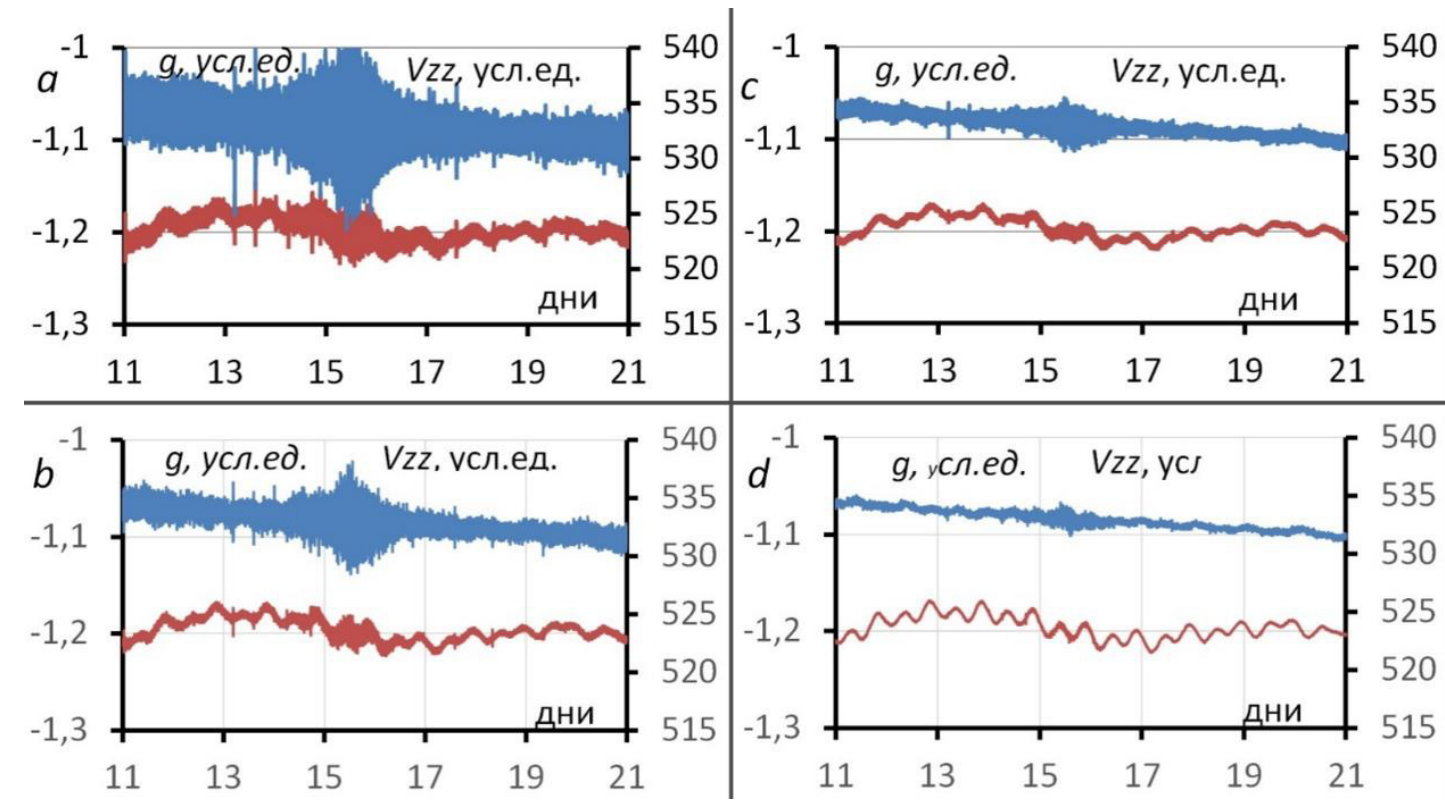

Рис. 3. Распределение значений силы тяжести и вертикального градиента, полученных с помощью метода осреднения на интервалах 15, 30, 60 и 120 секунд те же обозначения, что на рис. 2.

[Fig. 3. Distribution of gravity and vertical gradient values obtained using the averaging method at intervals of 15, 30, 60, and 120 seconds the same notation as in fig. 2.]

отмеченному выше, при вычислении градиента влияние лунно-солнечных вариаций минимально. Другое дело при вычислении силы тяжести. Здесь из исходных наблюдений вычитаются расчетные лунно-солнечные вариации силы тяжести, а в случае осреднения - среднеинтервальное значение. Но, расхождение между осредненным и истинным значениями вариаций легко оценить. Для этого достаточно расчетные вариации осреднить с нужным интервалом и вычесть из исходной вариации. На рис. 4 представлены такие расчеты для интервала 60 сек. Амплитуда расхождения невелика ( \pm 0.003 мГал), но она больше погрешности измерений и должна учитываться. Кроме того, следует отметить, что поправка сдвинута к исходной вариации: максимальные поправки приурочены к максимальным значениям градиента лунно-солнечных вариаций. 


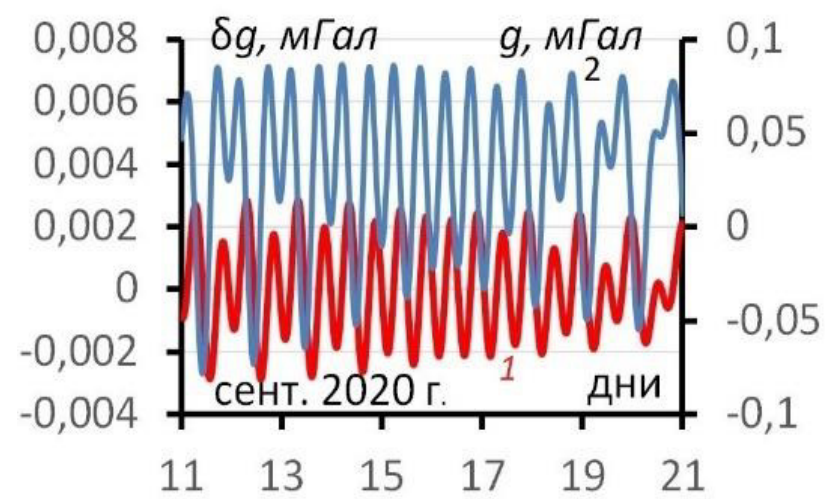

Рис. 4. Учет солнечных вариации при разделении наблюдений с помощью метода осреднения: $1-\delta \mathrm{g}$, разность между осредненными и исходными лунно-солнечными вариациями; 2 лунно-солнечные вариации.

[Fig. 4. Accounting for solar variations when dividing observations using the averaging method: $1-\delta \mathrm{g}$, the difference between the averaged and the original lunar-solar variations; 2 - lunar-solar variations.]

После проведения всех вычислений рассмотрим, как изменяются спектральные характеристики. Начнем с вычисления спектра для вертикального градиента, как наименее зависимой величины от лунно-солнечных вариаций. На рис. 5 представлен спектр за первые трое суток измерений градиента с шагом наблюдения в 1 сек (рис.1, b, кривая 1). На периодограмме и в частотном спектре (рис. 1) четко выделяются пятисекундный максимум и полу- и суточная волны [8]. Графики представлены в частотной и временной формах. Разницы никакой. В практическом аспекте низкочастотную часть лучше представлять во временной форме и наоборот. Можно предположить, что причиной полуи суточной волн является давление солнечного ветра [9]. Пятисекундный максимум можно легко объяснить собственными колебаниями атмосферы $[6,9]$.

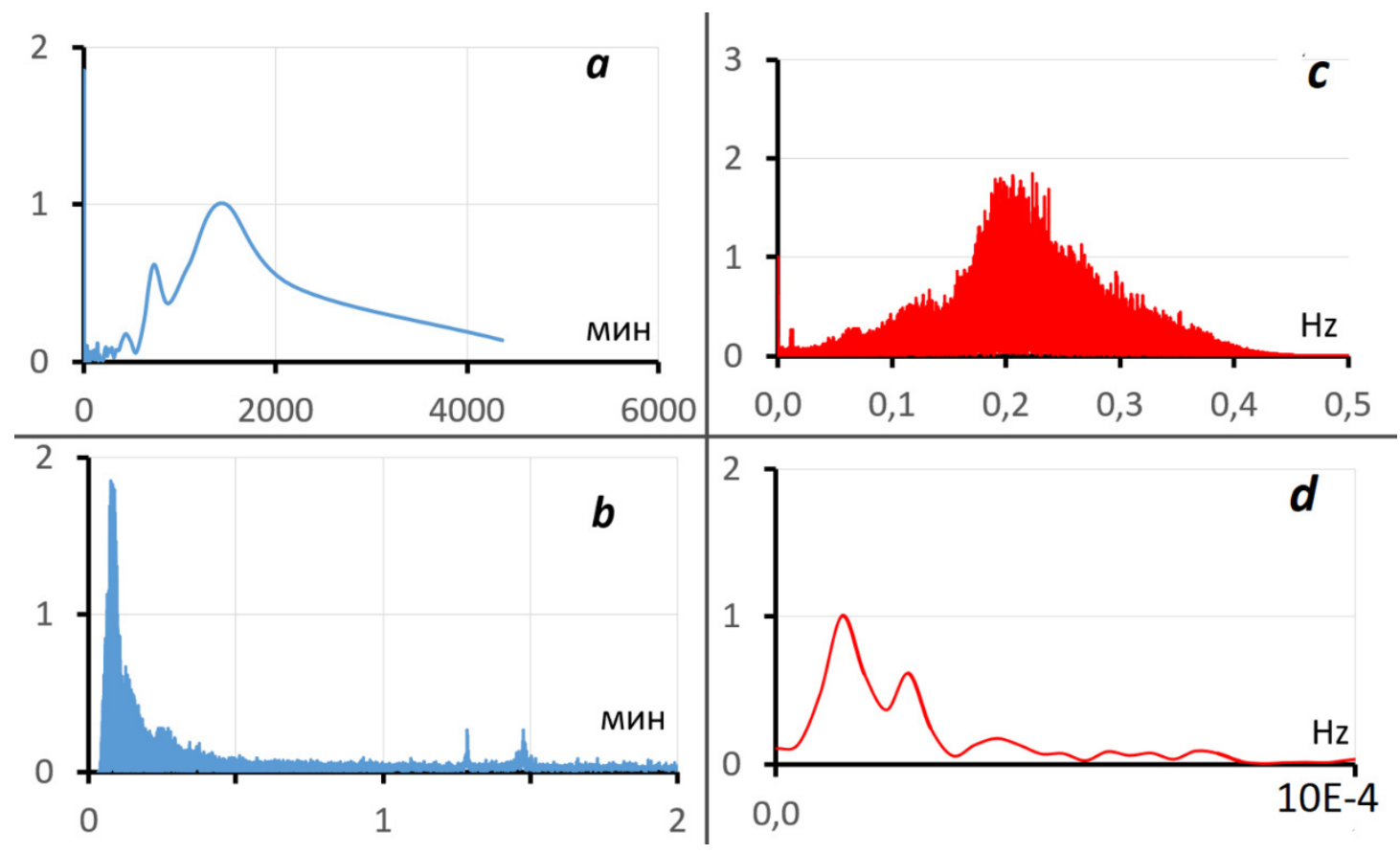

Рис. 5. Представление спектра с помощью периодограммы и в частотной форме: $a$ - периодограмма спектра в интервале $0-$ 5000 мин; $\mathrm{b}$ - периодограмма спектра в интервале 0 - 2 мин; с - спектр в интервале $0-0,5$ Герц (Hz); $\mathrm{d}$ - спектр в интервале 0 - $10 \mathrm{E}-4$ Герц $(\mathrm{Hz})$.

[Fig. 5. Representation of the spectrum using the periodogram and frequency: $a$ - periodogram of the spectrum in the range of $0-5000$ min; $b$ - periodogram spectrum in the range of -2 to $0 \mathrm{~min} ; c$ - spectrum in the range of $0-0.5 \mathrm{Hertz}(\mathrm{Hz}) ; d-$ range in the interval $0-10 \mathrm{E}-4$ Hertz (Hz).]

Увеличение амплитуды колебаний на исходных графиках (рис. $1, \mathrm{~b})$ также связаны с колебаниями атмосферы, которые увеличиваются за счет удара метеорного потока по атмосфере. Это легко проверяется путем вычисления спектров для разных частей графиков. Графики вертикального градиента и силы тяжести (рис.1, b) разделили на три части с учетом амплитуды колебаний, вычислили спектры отдельно для градиента и силы тяжести (рис. 6 и 7). Отмечается прямая зависимость между амплитудами колебаний и спектров, что еще раз свидетельствует о причинной связи между собственными колебаниями атмосферы и коле- баниями вертикального градиента и силы тяжести. Кроме того, такая же связь наблюдается между амплитудами полусуточной волны (рис. $6, d$ и $7, d$ ).

Теперь вычислим спектры для вертикального градиента и силы тяжести, полученных путем дискретной выборки и методом осреднения Спектры вычислены для всех вышеуказанных интервалов выборки, и они практически идентичны, поэтому приведем спектры лишь для интервала 60 с. Прежде всего отметим, что на спектрах для осредненных значений силы тяжести и ее вертикального градиента очень четко выделяются полу- и суточная волны (рис. $8, a, b$ ). 


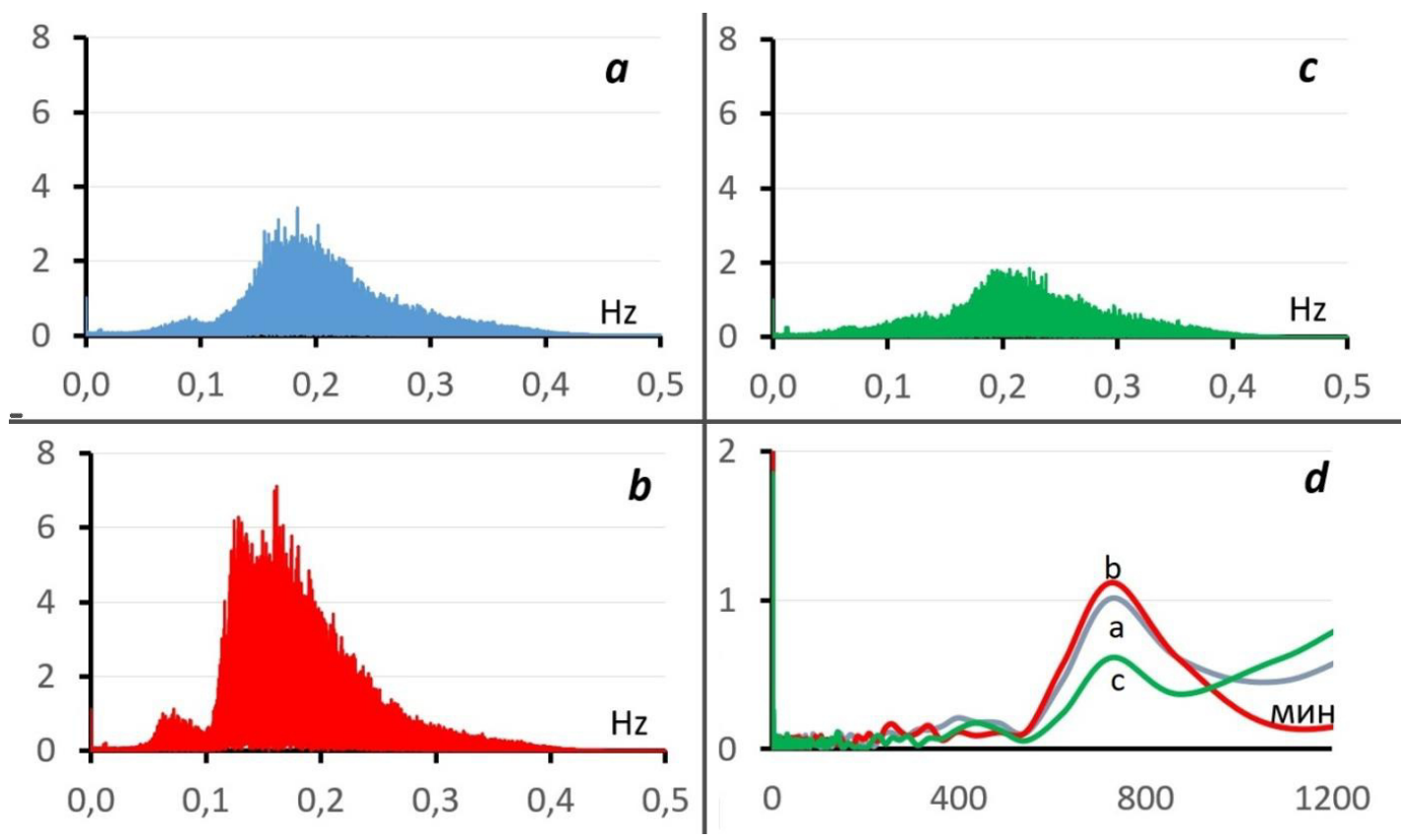

Рис. 6. Спектры для частей наблюдений с различной амплитудой колебаний, полученных с помощью осредненной выборки: $a, b, c$ - частотные спектры в герцах соответственно для интервалов со средней, максимальной и минимальной амплитудой колебаний; $d$ - изменение амплитуды сигнала полусуточной волны для тех интервалов во временной форме.

[Fig. 6. Spectra for observations with different amplitude, obtained using the averaged sample: $a, b, c$ - frequency spectra in Hertz, respectively, for intervals with average, maximum and minimum oscillation amplitudes; $d$ - change in the amplitude of the half-day wave signal for those intervals in time form.]

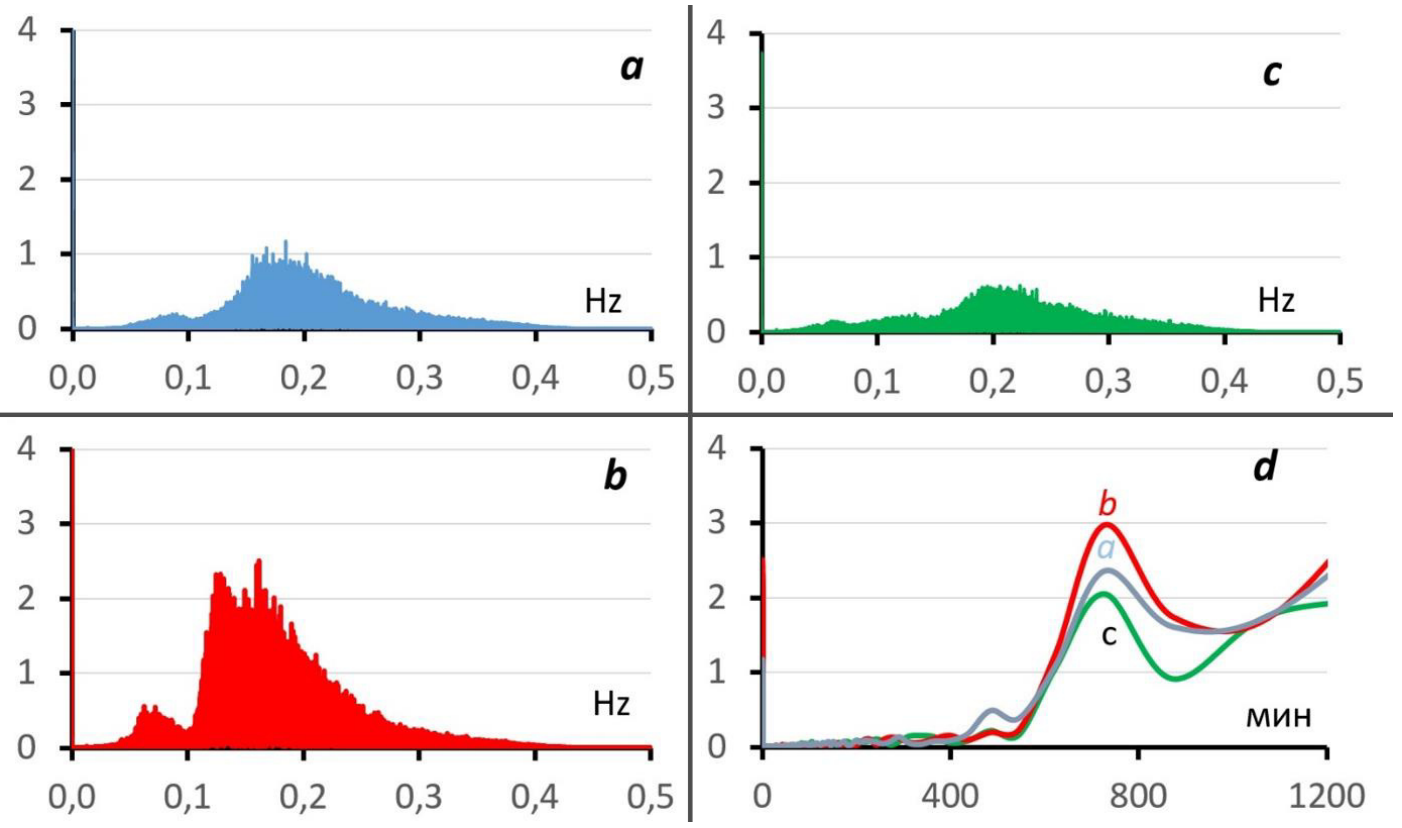

Рис. 7. Спектры для частей наблюдений с различной амплитудой колебаний, полученных с помощью дискретной выборки. Те же обозначения, что на рис. 6.

[Fig. 7. Spectra for parts of observations with different oscillation amplitudes obtained using a discrete sample. The same notation as in Fig. 6.]

Естественно, что пятисекундные максимумы исчезли даже при осреднении на интервалах в 15 сек. Что же касается дискретной выборки, то на периодограммах в обоих случаях (рис. $8, c, d$ ) никаких полезных сигналов не выделяется, даже полу- и суточной волн. Таким образом, осреднение при дискретизации является более предпочтительной трансформацией. Да, при осреднении часть информации пропадает [7], но если исключить осредненные данные из наблюденных значений, то в большей части восстановим информацию высокочастотной части. 


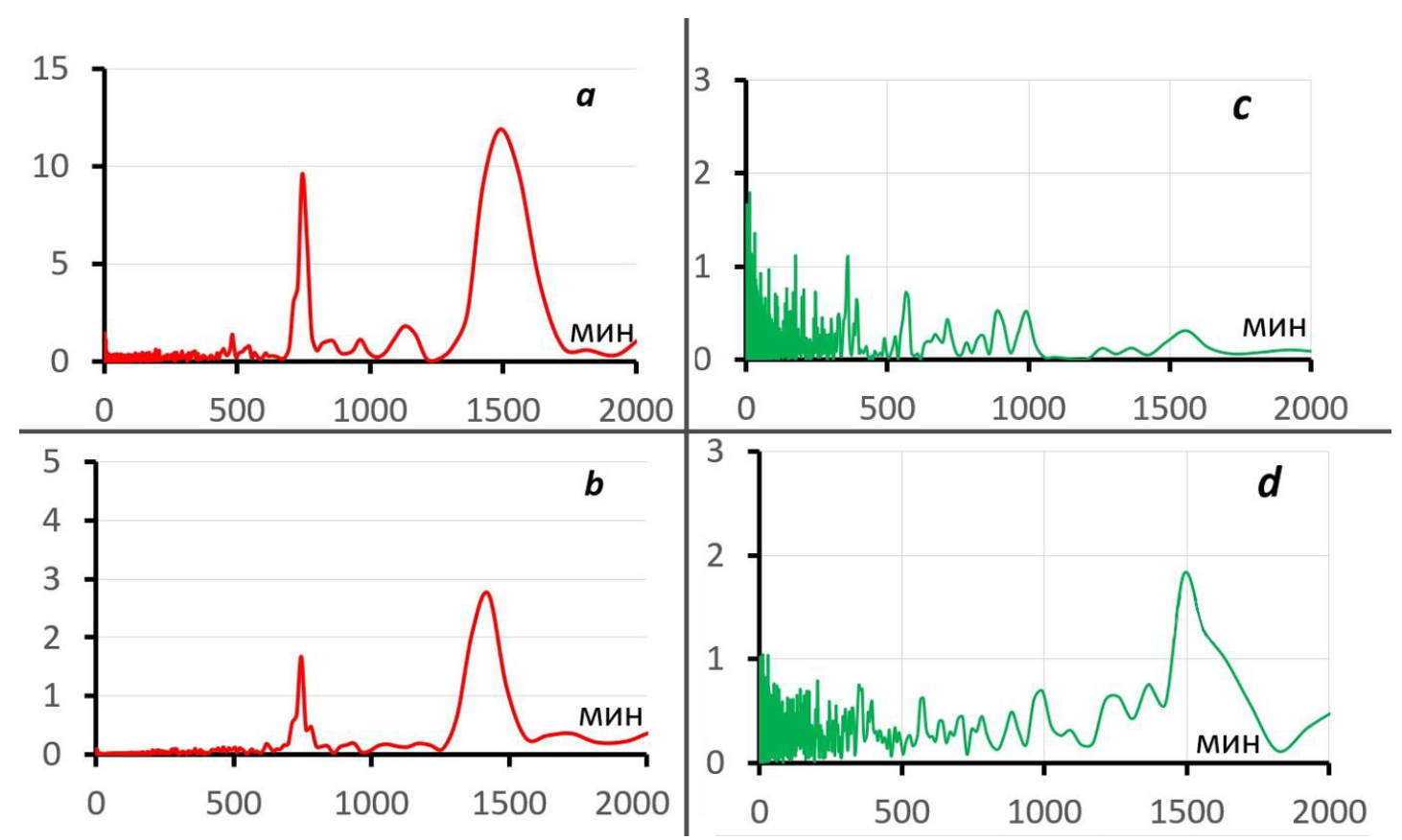

Рис. 8. Периодограммы спектрального анализа для дискретизации 60 с., полученные с помощью осреднения и дискретной выборки: $a$ - периодограмма спектра в интервале $0-2000$ мин вертикального градиента, полученного с помощью осреднения; $b-$ периодограмма спектра в интервале $0-2000$ мин силы тяжести полученной с помощью осреднения; $c$ - периодограмма спектра в интервале 0 - 2000 мин вертикального градиента, полученного с помощью дискретной выборки; $d$-периодограмма спектра в интервале 0 - 2000 мин силы тяжети полученной с помощью дискретной выборки.

[Fig. 8. Periodograms of spectral analysis for $60 \mathrm{~s}$ sampling obtained by averaging and sampling: $a$-periodogram of the spectrum in the range of $0-2000 \mathrm{~min}$ vertical gradient obtained by averaging; $b$-periodogram spectrum in the range of $0-2000$ min gravity obtained by averaging; $c$ - periodogram spectrum in the range of $0-2000$ min vertical gradient obtained with the discrete sampling; $d$-periodogram spectrum in the range of $0-2000 \mathrm{~min}$ power tagete obtained using discrete sampling.]

\section{Выводы}

В результате можно утверждать, что дискретизация с использованием осреднения сохраняет практически большую часть информации и должна использоваться при расчетах. Дискретизацию при простой выборке можно использовать преимущественно при качественной интерпретации результатов измерений. И наконец, следует отметить, что использование гравиметра с двумя датчиками внесет существенный вклад в изучение влияния космических и наземных факторов при изменении силы тяжести и ее вертикального градиента. В прикладном плане такие приборы внесут определенные коррективы в методику высокоточных измерений силы тяжести.

Конфликт интересов: Авторы декларируют отсутствие явных и потенциальных конфликтов интересов, связанных с публикацией настоящей статьи.

\section{ЛИТЕРАТУРА}

1. Project IDA [Электронный pecypc]. URL: https://sur.ly/i/ida.ucsd.edu/ (дата обращения: 1.11.2020)

2. Goodkind J.M The superconducting Gravimeter // Rev. Sci. Instrum. 1999. Vol. 70. No. 11. pp. 4131-4152.
3. Антонов Ю. В. Вычисление вертикального градиента силы тяжести по измерениям двухсферного сверхпроводящего гравиметра // Вестник Воронежского государственного университета. Серия: Геология. 2020. № 3. С. 55-63. DOI: https://doi.org/10.17308/geology.2020.3/3009

4. Антонов Ю. В. Пульсации неприливных вариаций силы тяжести // Известия высших учебных заведений. Геология и разведка. 2014. № 5. С. 54-57.

5. Антонов Ю. В., Сизаск И. А. Синхронные пульсации в неприливных вариациях гравитационного и сейсмического полей // Известия высших учебных заведений. Геология и разведка. 2015. №5. C. 46-52. DOI: https://doi.org/10.32454/0016$7762-2015-5-46-52$

6 Антонов Ю.В. Влияние атмосферного фронта на показания гравиметров и сейсмометров // Известия высших учебных заведений. Геология и разведка. 2017. №4. С. 66-1. DOI: https://doi.org/10.32454/0016-7762-2017-4-66-71

7. Антонов Ю. В. Разделение неприливных вариаций силы тяжести на основе спектрального анализа и метода осреднения // Вестник Воронежского государственного университета. Серия: Геология. 2016. № 2. С. 100-106.

8. Melchior H. J. The Earth's tides / Pergamon press.1968. 458 p. 9. Матвеев Л.Т. Физика атмосферы / Л.: Гидрометеоиздат , $2000.780 \mathrm{c}$. 
UDC 550.831

DOI: https://doi.org/10.17308/geology.2020.4/3127

ISSN 1609-0691

Received: 24.11 .2020

Accepted: 01.12.2020

Published online: 18.12 .2020

\title{
Processing the measurements from stationary gravimeters
}

\author{
Yu. V. Antonov ${ }^{\bowtie}$, I. Yu. Antonova, I. A. Ponomarenko \\ Voronezh State University 1 Universitetskaya pl., Voronezh 394018, Russian Federation
}

\begin{abstract}
Introduction: The comparison of gravitational waves at research units and observatories located on different continents demonstrated that the vibrations are synchronised. The measurements often vary in the degree of accuracy, which becomes obvious during their processing. In such cases, sampling has to be performed in order for the measurements from different units to have the same sampling interval. Most often, the most accurate measurement is determined, and the rest of the measurements are equated to this one. The same happens when there are too many measurements for computers to process. In this case, the accuracy of measurements can be improved by increasing the sampling interval. It can also be done by selecting individual values within a certain interval, or by calculating the mean interval value.

Methodology: Both methods were tested using the measurements of a dual sensor superconducting gravimeter, which allowed the evaluation of both the selection of gravity values and the vertical gravity gradient. We used the dual sensor superconducting gravimeter because at the moment it is one of the most accurate devices. The measurements were performed with a one second sampling interval, which enabled us to manually alter the sampling rate and assess the way its affected the measurements. The initial measurements provided by the device were taken from open sources.

Results and discussion: Since averaged interval sampling helps to retain most of the data, we suggest that it should be considered the optimal method for processing the measurements. Simple sampling can be used for qualitative interpretation of the measurements.

Conclusions: The experiment demonstrated that the optimal processing method is the averaged interval sampling.

Keywords: lunar-solar gravity variations; non-tidal gravity variations; vertical gravity gradient; meteor showers; pulsations.

For citation: Antonov Yu. V., Antonova I. Yu., Ponomarenko I. A. Processing the measurements from stationary gravimeters. Vestnik Voronezhskogo gosudarstvennogo universiteta. Seriya: Geologiya = Proceedings of Voronezh State University. Series: Geology, 2020, no. 4, pp. 59-66. DOI: doi.org/10.17308/geology.2020.4/3127
\end{abstract}

Conflict of interest: The authors declare the absence of obvious and potential conflicts of interest related to the publication of this article.

\section{REFERENCES}

1. Project IDA. Available at: https://sur.ly/i/ida.ucsd.edu/ (accessed 1.11.2020)

2. Goodkind J.M The superconducting Gravimeter, Rev. Sci. Instrum. 1999, vol. 70, no. 11, pp. 4131-4152.

3. Antonov $\mathrm{Yu}$. V. Calculation of the vertical gravity gradient using the measurements of a dual-sphere superconducting gravimeter. Vestnik Voronezhskogo gosudarstvennogo universiteta. Seriya: Geologiya = Proceedings of Voronezh State University.
Series: Geology, 2020, no. 3, pp. 100-106. (In Russ.). DOI: https://doi.org/10.17308/geology.2020.3/3009

4. Antonov Yu.V. Pul'satsii neprilivnykh variatsiy sily tyazhesti [Ripple tidal gravity variations]. Izvestiâ vysših učebnyh zavedenij. Geologia i razvedka = Proceedings of higher educational establishments. Geology and Exploration, 2014, no. 5, pp. 54-57.2. (In Russ.)

5. Antonov Yu. V, Sizask I. A. Sinkhronnye pul'satsii v neprilivnykh variatsiyakh gravitatsionnogo i seysmicheskogo poley

The content is available under Creative Commons Attribution 4.0 License.

\footnotetext{
$\bowtie$ Yuriy V. Antonov, e-mail: yuriyantonov@yandex.ru
} 
[Synchronous ripples innon-tidal variations of gravitational and seismicfields]. Izvestiâ vysših učebnyh zavedenij. Geologiâ $i$ razvedka $=$ Proceedings of higher educational establishments. Geology and Exploration, 2015, no. 5, pp. 46-52. (In Russ.). DOI: https://doi.org/10.32454/0016-7762-2015-5-46-52

6. Antonov $\mathrm{Yu}$. V. Vliyanie atmosfernogo fronta na pokazaniya gravimetrov i seymometrov [Influence of the atmospheric front on the readings of gravimeters and seismometers]. Izvestiâ vysših učebnyh zavedenij. Geologia $i$ razvedka $=$ Proceedings of higher educational establishments. Geology and Exploration, 2017, no.
4, pp. 66-71. (In Russ.). DOI: https://doi.org/10.32454/00167762-2017-4-66-71

7. Antonov $\mathrm{Yu}$. V. Separation of non-tidal gravity variations based on spectral analysis and averaging method. Vestnik Voronezhskogo gosudarstvennogo universiteta. Seriya: Geologiya $=$ Proceedings of Voronezh State University. Series: Geology, 2013, no. 2, pp. 100-106. (In Russ.).

8. Melchior H.J. The Earth's tides. Pergamon press.1968. 458 p. 9. Matveev L.T. Phyzika atmosfery [Atmospheric physics]. Gidrometeoizdat publ., 2000, 780 p.
Антонов Юрий Васильевич - д. тех. н., профессор, Воронежский государственный университет, Воронеж, Российская Федерация; E-mail: yuriyantonov@yandex.ru; ORCID http://orcid.org/0000-0002-3323-9697

Антонова Ирина Юрьевна - преподаватель, Воронежский государственный университет, Воронеж, Российская Федерация; E-mail: mavka_r@mail.ru;

ORCID http://orcid.org/0000-0001-5851-3122

Пономаренко Иван Александрович - аспирант кафедры геофизики, Воронежский государственный университет, Воронеж, Российская Федерация; E-mail: kochuma@yandex.ru; ORCID http://orcid.org/0000-0002-2588-3917

Авторы прочитали и одобрили окончательный вариант рукописи.
Yuriy V. Antonov - Dr.habil. in Techn., Professor, Voronezh State University, Voronezh, Russian Federation; E-mail: yuriyantonov@yandex.ru; ORCID http://orcid.org/0000-0002-33239697

Irina Yu. Antonova - Lecturer, Voronezh State University, Voronezh, Russian Federation; E-mail: mavka_r@mail.ru; ORCID http://orcid.org/0000-0001-5851-3122

Ivan A. Ponomarenko - postgraduate student, Voronezh State University, Voronezh, Russian Federation;

E-mail: kochuma@yandex.ru;

ORCID http://orcid.org/0000-0002-2588-3917

The authors have read and approved the final manuscript. 